\title{
Association among Soluble Tumor Necrosis Factor Receptor I, Matrix Metalloproteinase-9, Procollagen C-terminal Propeptide type I, and N-Terminal proBrain Natriuretic Peptide in Heart Failure
}

\author{
Mona Yolanda ${ }^{1,2^{*}}$, Marsetio Donosepoetro ${ }^{3}$, Anwar Santoso $^{4}$, Mansyur Arief $^{1}$ \\ ${ }^{1}$ Post Graduate Program in Clinical Biochemistry, Hassanudin University, Jl. Perintis Kemerdekaan Kav. 10, Makassar, Indonesia \\ ${ }^{2}$ Prodia Clinical Laboratory, Jl. Cisangkuy No.2, Bandung 40114, Indonesia \\ ${ }^{3}$ Faculty of Medicine, Airlangga University, Jl. Mayjen Prof. Dr. Moestopo No. 47, Surabaya, Indonesia \\ ${ }^{4}$ National Cardiovascular Center-Harapan Kita, JI. Letjen. S. Parman Kav 87, Slipi, Jakarta, Indonesia \\ 'Correspondence: mona.yolanda@prodia.co.id
}

\section{Abstract}

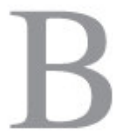

ACKGROUND: Heart failure (HF) is associated with an increased expression of proinflammatory cytokines, especially soluble tumor necrosis factor receptor I (sTNFR I), but the underlying mechanism to the relationship between sTNFR I activation and the progression of HF is not yet fully understood. This study aims to see the association between sTNFR I, MMP-9, PICP, and NT-proBNP in the progression of HF.

METHODS:Thiswasacrosssectionalstudywhichrecruited 45 subjects with HF confirmed by echocardiography and NT-proBNP. Concentration sTNFR I, MMP-9, and PICP were measured using ELISA method, whereas NT-proBNP concentration was measured using ECLIA method. Univariate linear regression analysis, path analysis and General Linear Model were used to determine which parameters played the most significant role in HF.

RESULTS: Results of the univariate linear regression and path analysis showed there was a linear relationship between sTNFR I with MMP-9, with R square of $25.8 \%$ $(\mathrm{p}=0.00 ; \mathrm{r}=0.508)$, $\mathrm{R}$ square $\mathrm{sTNFRI}$ and MMP-9 with PICP was $14.4 \%(p=0.038 ; r=0.379)$ and $R$ square MMP-9 and PICP with NT-proBNP was 39.6\% ( $\mathrm{p}=0.00$; $r=0.629$ ). From the General Linear Model we found that the important predictor for HF was through MMP-9 and PICP.
CONCLUSION: sTNFR I as a proinflammatory factor is one of the factors involved in the heart failure as seen by NT-proBNP through activation of fibrosis (PICP) and remodeling factor (MMP-9).

KEYWORDS: sTNFR I, MMP-9, PICP, NT-proBNP, heart failure.

Indomes Biomed J 2011: 3(1): 64-69

\section{Introduction}

Heart failure is seen as a complex situation due to changes in the structure and function of the myocardium as well as activation of several neurohormonal systems that have an effect on this process. Neurohormonal activation complex aims to improve the mechanical condition of the cardiac hormone, such as circulation and local pro-inflammatory cytokine that plays an important role in disease progression (1).

Heart failure is associated with an increased expression of proinflammatory cytokines, especially TNF- $\alpha$ (tumor necrosis factor- $\alpha$ ), but the underlying mechanism of the relationship between TNF- $\alpha$ activation and the progression of the heart failure is not yet fully understood. Previous studies consistently showed a direct relationship between concentrations of cytokines and worsening of the heart 
failure (2). In this study we used sTNFRI, which is a TNF- $\alpha$ receptor, as a marker for pro-inflammatory cytokines.

The matrix metalloproteinases(MMPs) are proteinases that play a role in the degradation of extracellular matrix. This study using MMP-9 had a significant contribution to explain the role in cardiovascular disease processes involved in vascular remodeling, atherosclerotic plaque instability, and left ventricular remodeling after myocardial infarction (3).

Fibrosis contributes to rigidity. Myocardial fibrosis would disrupt the coordination of excitation-contraction in both diastolic and systolic. In this study biochemical marker PICP (C-terminal collagen type I Propetide) was used as a biochemical marker for collagen synthesis (4).

The American College of Cardiology Foundation/ American Heart Association Practice Guidelines : Focus Update 2009 described the determination of heart failure according to New York Heart Association (NYHA, and examination of the role of B-type natriuretic peptide (BNP) and N-terminal pro B-type natriuretic peptide (NTproBNP), a comprehensive evaluation on heart failure patients. This guide explained that the examination of NTproBNPand BNP is recommended for clinical determination of patients who showed symptoms of heart disease. This guide explains that the examination of NT-proBNP and BNP is recommended for clinical determination of patients who showed symptoms of heart disease. Measurement of natriuretic peptides (BNP and NT-proBNP) is useful in the evaluation of patients in emergency situations with a clinical diagnosis of heart failure being unclear for risk stratification by the level of evidence A class (5).

The study was conducted to see the role of proinflammatory cytokine that induces fibrosis and remodelling factors in heart failure.

\section{Methods}

Forty five patients with heart failure, aged above 40 years were enrolled in this cross sectional study. Participants signed informed consents to have personal data such as height, weight, serum measurements, blood pressure, and waist circumference. The study's proposal was approved by The Health Research Ethics Committee of The Faculty of Medicine Hasanuddin University of Makassar and Udayana University of Denpasar.
sTNFR I (cat\#SRT100) and MMP-9 (cat\#DMP900) were measured by ELISA method, Enzyme Linked Immunosorbent Assay kit (R\&D Systems), PICP (cat\#8003) was measured by Enzyme Immunoassay kit $\left(\right.$ Quidel ${ }^{\mathrm{R}}$ ), and NT-proBNP (cat\#04842464) was measured by electrochemiluminescence immunoassay (Roche). Sera were separated from whole blood specimens after centrifugation, and immediately kept at $-20^{\circ} \mathrm{C}$ until assayed. All assays were performed according to manufacturers' instructions.

Statistical analyses were performed with the SPSS for Windows version 13 (SPSS Inc., Chicago, IL, USA). Distributions of continuous variables were assessed for normality using the Kolmogorov-Smirnov. Using univariate linear regression, path analysis and general liniar model were used to see the interaction of various variables on heart failure to determine the most influential variable on the heart failure. The results are presented in forms of narration and tables and graphs. For statistical tests, we set the level of significance at $5 \%$.

\section{Results}

Table 1 describes the clinical and biochemical characteristics of the subjects. Table 2 and 3 showed correlation between variables and general linear model analysis of sTNFR I, MMP-9, PICP, and NT-proBNP. Figure 1 showed path analysis of sTNFR I, MMP-9, PICP, and NT-proBNP in heart failure.

The path analysis showed that there was a linear relationship between sTNFR I with MMP-9, R square was $25.8 \%(p=0.00 ; r=0.508)$, R square sTNFR $I$ and MMP-9 with PICP was $14.4 \%(\mathrm{p}=0.038 ; \mathrm{r}=0.379)$ and $\mathrm{R}$ square MMP-9 and PICP with NT-proBNP was $39.6 \%$ (p $=0.00 ; r=0.629)$. From all of the variable showed that the influence sTNFR I, MMP-9 and PICP to NT-proBNP of $61.7 \%$ and the influence of other variables that are not obtained from the model for $38.3 \%$. Heart failure (based on NT-proBNP) more strongly influenced by inflammatory factors (sTNFR I) through a path of factor remodeling (MMP-9) and the fibrosis (PICP).

Figure 1 showed path analysis of sTNFR I, MMP-9, PICP, and NT-proBNP in heart failure. 


\section{Table 1. Clinical and Biochemical Characteristics of Heart Failure}

\begin{tabular}{lc}
\hline Variables & Mean \pm SD \\
\hline Age (years) & $61.4 \pm 9.2$ \\
WC (cm & $89.1 \pm 9.0$ \\
SBP $(\mathrm{mmHg})$ & $120.9 \pm 17.4$ \\
DBP $(\mathrm{mmHg})$ & $77.6 \pm 10.7$ \\
EF (\%) & $61.3 \pm 18.7$ \\
SGOT (U/L) & $26.5 \pm 14.7$ \\
SGPT (U/L) & $26.0 \pm 20.3$ \\
FPG (mg/dL) & $109.5 \pm 38.6$ \\
Total Cholesterol (mg/dL) & $191.0 \pm 36.5$ \\
Triglyceride (mg/dL) & $120.3 \pm 48.8$ \\
HDL Cholesterol (mg/dL) & $46.1 \pm 9.2$ \\
LDL Cholesterol (mg/dL) & $125.8 \pm 31.9$ \\
CCT (mL/min/1.73m²) & $48.8 \pm 19.1$ \\
hsCRP (mg/dL) & $2.2 \pm 2.8$ \\
sTNFR I (pg/mL) & $2.2 \pm 1.1$ \\
NT-proBNP (pg/mL) & $18.8 \pm 1.4$ \\
MMP-9 (ng/mL) & $495.2 \pm 243.4$ \\
PICP (ng/mL) & $73.5 \pm 24.3$ \\
\hline
\end{tabular}

Description: $\mathrm{WC}=$ Waist Circumference; $\mathrm{SBP}=$ Systolic Blood Pressure; DBP= Diastolic Blood Pressure; $\mathrm{EF}=$ Ejection Fraction; SGOT = Serum Glutamic Oxalacetatic Transaminase; SGPT = Serum Glutamic Piruvate Transaminase; FPG = Fasting Plasma Glucose; HDL = High Density Lipoprotein; LDL = Low Density Lipoprotein; $C C T=$ Creatinine Clearance Test; hs-CRP $=$ high sensitivity C-Reactive Protein; sTNFR I = soluble Tumor Necrosis Factor Receptor I; NT-proBNP = N-Terminal-pro Brain Natriuretic Peptide; MMP-9 = Matrix Metalloproteinase-9; PICP = Procollagen C-terminal Propeptide type I; SD= Standard Deviation.

Table 2.Correlation between variables

\begin{tabular}{lcc}
\hline Variables & $\mathbf{r}$ & $\mathbf{P}$ \\
\hline STNFR I & $0.474^{* *}$ & 0.001 \\
MMP-9 & $0.537^{* *}$ & 0.000 \\
PICP & $0.488^{* *}$ & 0.001 \\
\hline
\end{tabular}

Description : sTNFR I = soluble Tumor Necrosis Factor Receptor I; MMP-9 = Matrix Metalloproteinase-9; PICP = Procollagen C-terminal Propeptide type I; NT-proBNP = N-Terminal-pro Brain Natriuretic Peptide. 
Table 3. Univariate Linear Regression and GLM Univariate Analysis

\begin{tabular}{lccccccc}
\hline Variables & B & Coefficient B & SE & T & p & \multicolumn{2}{c}{$95 \%$ C.I } \\
& & & & & & Lower & Upper \\
\hline STNFR I & 1.098 & 0.206 & 0.747 & 1.471 & 0.149 & -0.41 & 2.606 \\
MMP-9 & 0.001 & 0.329 & 0.001 & 2.342 & 0.024 & 0.00 & 0.002 \\
PICP & 0.011 & 0.312 & 0.005 & 2.44 & 0.019 & 0.002 & 0.021 \\
\hline
\end{tabular}

Description : sTNFR I = soluble Tumor Necrosis Factor Receptor I; MMP-9 = Matrix Metalloproteinase-9; PICP = Procollagen C-terminal Propeptide type I; NT-proBNP = N-Terminal-pro Brain Natriuretic Peptide.

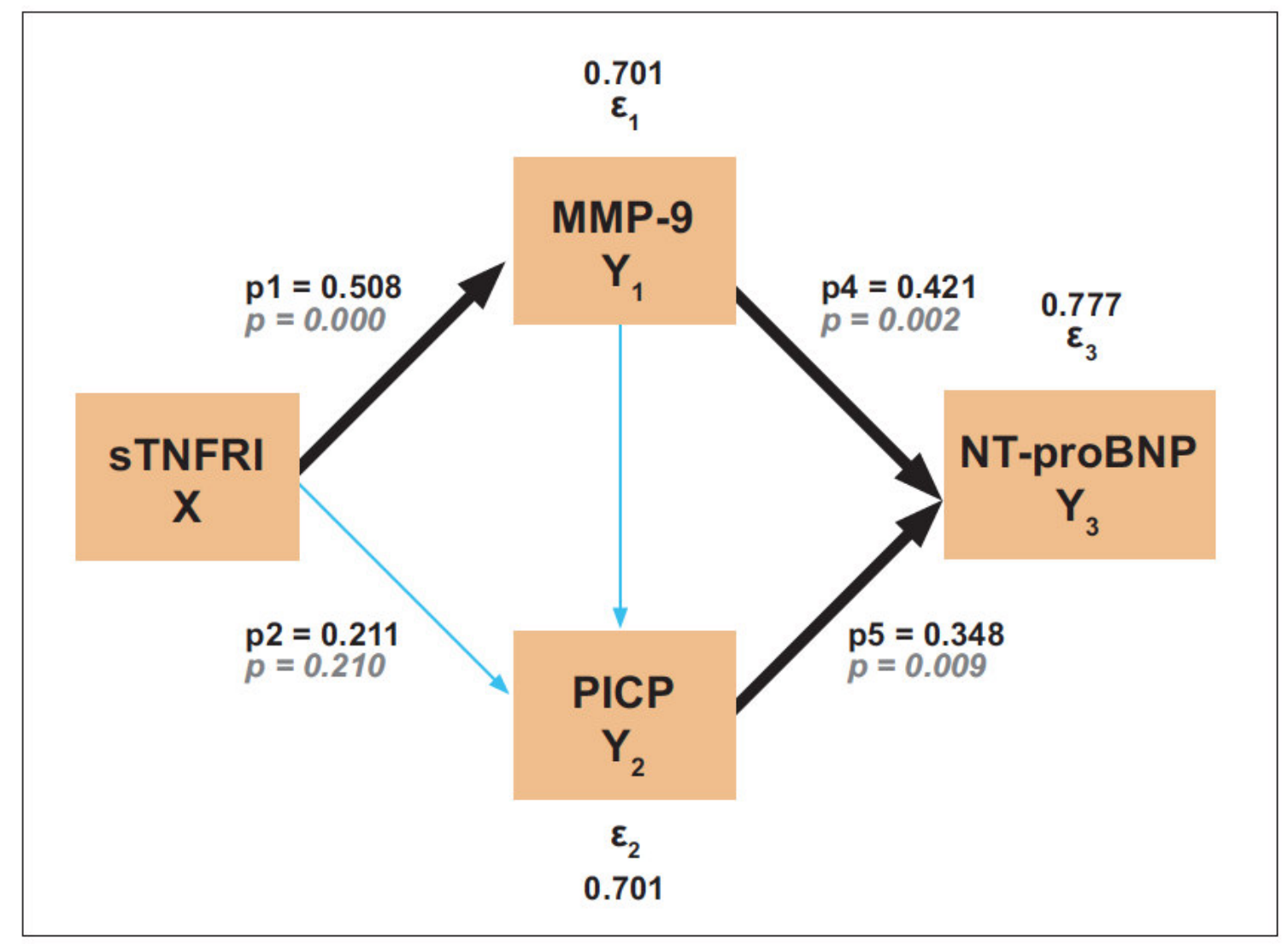

Figure 1. Path analysis Diagram. It appears from the drawing thick lines indicate significant pathways that can worsen the condition of heart failure. 


\section{Discussion}

\section{Analysis of Correlation between sTNFR I, MMP-9, PICP, and NT-proBNP in heart failure}

This study showed that there was a significant positive correlation between STNFR I with MMP-9 $(r=0.508 * *$, $\mathrm{p}=0.000)$, and PICP $(\mathrm{r}=0.326 * \mathrm{p}=0.029)$. It was clear that sTNFR I induced the secretion PICP and MMP-9, which associated with the development of heart failure.

Inflammation is important in the pathogenesis and progression of many forms of heart failure, and biomarkers of inflammation have become the subject of intense inquiry. According to the cytokine hypothesis of heart failure, proinflammatory cytokines (TNF- $\alpha$, IL-1, IL-6, dan IL-18) are produced by the damaged myocardium; this production is enhaced by stimulation of the sympathetic nervous system. Injured myocardium, as well as skeletal muscle that is hypoperfused because of reduced cardiac output activates monocytes to produce the same cytokines, which act on and further impair myocardial function. The stressed myocardium releases natriuretic peptides to improve heart function. Expression of pro-inflammatory cytokines which exacerbate the effects associated with left ventricular function and accelerate the progression of heart failure (6).

Left ventricular (LV) remodeling is an important contribution event in the progression to end-stage congestive heart failure (CHF). MMPs and TIMPs are regulated at the transcriptional level by binding of transcription factors, enhancers and repressors to the gene's promoter region and their expression is modulated by external stimuli that are operative in cardiac disease states. MMP-9 has a nuclear factor- $x \mathrm{~B}$ (NF- $x \mathrm{~B})$ binding site $(7,8)$.

Binding of TNF induces the trimerization of the TNFR and the recruitment of a number of adaptor proteins. Kawamura et al. examined the effects of a double transgenic mouse model in which cardiomyopathy was induced by cardiac restricted overexpression of TNF and transcription was altered due to a disrupted p50 NF$\chi \mathrm{B}$ subunit. With TNF overexpression only, MMP-9 was increased. However, with the TNF overexpression and disrupted NF- $x$ B, the levels of MMP- 9 were significantly attenuated (7).

It is accepted that myocardial fibrosis is the result of both increased collagen synthesis by fibroblasts and myofibroblasts and unchanged or decreased extracellular collagen degradation (9).

It is accepted that myocardial fibrosis is the result of both increased collagen synthesis by fibroblasts and myofibroblasts and unchanged or decreased extracellular collagen degradation (9).

Changes in the quantity and quality of the collagen network leads to changes in the structure and function of the left ventricle. Increased collagen, as shown in hypertrophy due to excessive pressure eventually increases stiffness of the wall of the myocardium and causes left ventricular hypertrophy. While degradation of the existing collagen as in myocardial infarction, reduces stiffness in the wall of the heart muscle and causes dilatation of the left ventricle. So both will lead to the abnormalities in left ventricular function $(10,11)$.

Fibrotic tissue accumulation is an integral part of structural changes in heart tissue. PICP is Carboxyterminal propeptide of type I and associated with collagen deposition in the myocardium in heart failure patients $(9,12)$.

Univariate linear regression, path analysis, and general linear model of STNFR I, MMP-9, and PICP in heart failure

From univariate linear regression and general linear model, we found that the greatest role of factors in heart failure was that of PICP and MMP-9, and based on path analysis the overall influence level of sTNFR I, MMP-9, PICP in the state of heart failure was $61.7 \%$ and that of the other factors outside the model was $38.3 \%$.

Remodeling of extracellular matrix in the early stages of the process leading to heart failure. Collagen accumulation increased with the progression of hypertrophic changes in spite of enhanced MMP levels. It is not clear which event was initial, reactive fibrosis or MMP activation, as both were already present in patients with compensated hypertrophy and could be provoked by the same factors $(13,14)$.

According to the study results based on this model, TNFR I induces MMP-9 as a factor in the remodeling and then the process of fibrosis supervened characterized by an increase in PICP. MMP may participate in fibrosis and remodeling process through digestion of matrix component and regulation of the formation matrikines such as glycil-histidyl-lysine and release of biologically active factors from the ECM (including TGF $\beta 1$, insulinlike growth factor, and fibroblast growth factor). Increase of sTNFRI, MMP-9 and PICP concentrations showed a poor prognosis in heart failure (15).

We realize that this study, however, does have limitations. In example, lack of consideration to subjects smoking, exercise and drug habits. Thus, a more advanced studies are needed, with a larger number of subjects; compared with a control groups; and also with attention to subjects smoking, exercise and drug habits. 


\section{Discussion}

From our study there were some important points. The prognosis of heart failure can be seen through examination of sTNFR I which is a receptor of TNF- $\alpha$ as a proinflamatory cytokines that cause remodeling factors (MMP-9) and fibrosis (PICP). Higher the concentration of each, referred to worse prognosis. From univariate linear regression and general linear model, MMP-9 and PICP may play roles in the association between STNFR I and the increased risk of HF.

\section{Acknowledgements:}

We thank The Prodia Education and Research Institute and Sanglah Hospital Denpasar for collecting samples.

\section{References:}

1. Mair J. Pathophysiology and Laboratory Medicine, Pathophysiology of Heart Failure. In Cardiac Biochemical Markers, Wu, A.H.B, eds., Totowa New Jersey : Humana Press, 2 nd, 2003: 351-67.

2. Murray DR, Freeman GL. Proinflammatory Cytokines : Predictors of a Failing Heart. Circulation 2003; 107: 1460-2.

3. Visse R, Nagase, H. Matrix Metalloproteinases dan Tissue Inhibitors of Metalloproteinases: Structure, Function, and Biochemistry. Circ Res 2003; 92: 827-39.

4. Cuspidi C, Ciulla M, Zanchetti A. Hypertensive Myocardial Fibrosis. Nephrol Dial Transplant 2006; 21: 20-3.
5. Jessup M, Abraham WT, Casey DE, Feldman AM, Francis GS, Ganiats TD, et al. 2009 Focused Update : ACC/AHA Guidelines for the Diagnosis and Management of Heart Failure in Adults. Circulation 2009; 119: 1977-2016

6. Braunwald E. Biomarkers in Heart Failure. N Engl J Med 2008; 358: $2148-59$.

7. Deschamps AM, Spinale FG. Extracellular Matrix : In Molecular Mechanism of Cardiac Hypertrophy and Failure. Walsh, R.A., 2005; 7:125-146.

8. Spinale FG, Coker ML, Heung LJ, Bond BR, Gunasinghe HR, Etoh T, et al. A Matrix Metalloproteinase Induction/Activation System Exists the Human Left Ventricular Myocardium and Is Upregulated in Heart Failure. Circulation 2000; 102: 1944-9.

9. Garcia-Bolao I, Lopez B, Macias A, Gavira JJ, Azcarate P, Diez J. Impact of Collagen Type I Turnover on the Long-term Response to Cardiac Resynchronization Therapy. Eur Heart J 2008; 29: 898-906.

10. DeschampsAM,SpinaleFG. Pathway of Matrix Metalloproteinase Induction in Heart Failure: Bioactive Molecules and Transriptional Regulation. Cardiovasc Res 2006: 666-76

11. Lopez B, Gonzalez A, Diez J. Ciculating Biomarkers of Collagen Metabolism in Cadiac Disease. Circulation 2010; 121: 1645-54.

12. Zannad F, Alla F, Dousset B ,Perez A, Pitt B. Limitation of Excessive Extracellular Matrix Turnover May Contribute to Survival Benefit of Spironolactone Therapy in Patients with Congestive Heart Failure. Circulation 2000;102: 2700-6.

13. Hansson J, Lind L,Hulthe J,Sundstrom J. Relations of Serum MMP-9 and TIMP-1 Levels to Left Ventricular Measures and Cardiovascular Risk Factors: A Population-Based Study. Eur J Cardiovasc Prev Rehabil 2009: 16: 297-303.

14. Polyakova V, Hein S, Kostin S, Ziegelhoffer, Schaper J. Matrix Metalloproteinases and Their Tissue Inhibitors in PressureOverloaded Human Myocardium during Heart Failure Progression. J.Am.Coll.Cardiol 2004; 44: 1609-18.

15. Li YY, McTiernan CF, Feldman AM. Interplay of Matrix Metalloproteinases, Tissue Inhibitors of Metalloproteinases and Their Regulators in Cardiac Matrix Remodeling. Cardiovasc Res 2000: 46: 214-24. 